\title{
A proposal for the use of uniform diagnostic criteria for gestational diabetes in Europe: an opinion paper by the European Board \& College of Obstetrics and Gynaecology (EBCOG)
}

\author{
Katrien Benhalima $^{1}$ - Chantal Mathieu ${ }^{1}$ Peter Damm ${ }^{2}$ - André Van Assche ${ }^{3}$.

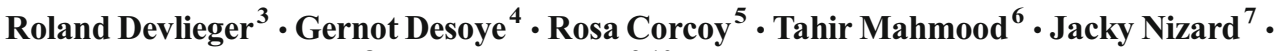 \\ Charles Savona-Ventura ${ }^{8} \cdot$ Fidelma Dunne $^{9,10}$
}

Received: 29 December 2014 / Accepted: 15 April 2015 / Published online: 8 May 2015

(C) Springer-Verlag Berlin Heidelberg 2015

\begin{abstract}
Screening and diagnostic criteria for gestational diabetes (GDM) are inconsistent across Europe, and the development of a uniform GDM screening strategy is necessary. Such a strategy would create opportunities for more women to receive timely treatment for GDM. Developing a consensus on screening for GDM in Europe is challenging, as populations are diverse and healthcare delivery systems also differ. The European Board \& College of Obstetrics and Gynaecology (EBCOG) has responded to this challenge by appointing a steering committee, including members of the EBCOG and the Diabetic Pregnancy Study Group (DPSG) associated with the EASD, to develop a proposal for the use of uniform diagnostic criteria for GDM in Europe. A proposal has been developed and has now been approved by the Council of the EBCOG. The current proposal is to screen
\end{abstract}

The authors were appointed by the EBCOG to form a steering committee to develop the current proposals.

\section{Katrien Benhalima}

katrien.benhalima@uzleuven.be

1 Department of Endocrinology, UZ Gasthuisberg, KU Leuven, Herestraat 49, 3000 Leuven, Belgium

2 Center for Pregnant Women with Diabetes, Department of Obstetrics, Juliane Marie Centre, Rigshospitalet, Clinical Institute of Medicine, Faculty of Health and Medicine Sciences, University of Copenhagen, Copenhagen, Denmark

3 Department of Obstetrics \& Gynecology, UZ Gasthuisberg, KU Leuven, Leuven, Belgium

4 Department of Obstetrics \& Gynecology, Medical University of Graz, Graz, Austria for overt diabetes at the first prenatal contact using cut-off values for diabetes outside pregnancy, with particular efforts made to screen high-risk groups. When screening for GDM is performed at 24 weeks' gestation or later, the proposal is now to use the $75 \mathrm{~g}$ OGTT with the new WHO diagnostic criteria for GDM. However, more research is necessary to evaluate the best GDM screening strategy for different populations in Europe. Therefore, no clear recommendation has been made on whether a universal one-step, two-step or a risk-factorbased screening approach should be used. The use of the same WHO diagnostic GDM criteria across Europe will be an important step towards uniformity.

Keywords Diagnostic criteria - Gestational diabetes · Proposal · Screening
5 Endocrinology and Nutrition Department, Hospital de la Santa Creu I Sant Pau, Barcelona, Spain

6 Department of Obstetrics \& Gynecology, University of Edinburgh, Edinburgh, UK

7 Department of Obstetrics \& Gynecology, AP-HP Groupe Hospitalier Pitié-Salpêtrière, Paris, France

8 Department of Obstetrics \& Gynecology, University of Malta Medical School, Msida, Malta

9 Endocrinology School of Medicine, National University of Ireland, Galway, Ireland

10 Galway Diabetes Research Centre, National University of Ireland, Galway, Ireland 


\begin{tabular}{|c|c|}
\hline \multicolumn{2}{|c|}{ Abbreviations } \\
\hline ACOG & $\begin{array}{l}\text { American College of Obstetricians and } \\
\text { Gynecologists }\end{array}$ \\
\hline DPSG & Diabetic Pregnancy Study Group \\
\hline EBCOG & $\begin{array}{l}\text { European Board \& College of Obstetrics and } \\
\text { Gynaecology }\end{array}$ \\
\hline FPG & Fasting plasma glucose \\
\hline GCT & Glucose challenge test \\
\hline GDM & Gestational diabetes \\
\hline HAPO & $\begin{array}{l}\text { Hyperglycaemia and Adverse Pregnancy } \\
\text { Outcome }\end{array}$ \\
\hline IADPSG & $\begin{array}{l}\text { International Association of Diabetes and } \\
\text { Pregnancy Study Groups }\end{array}$ \\
\hline LGA & Large for gestational age \\
\hline NIH & National Institutes of Health \\
\hline
\end{tabular}

\section{Screening for gestational diabetes}

The initial criteria for diagnosis of gestational diabetes (GDM) were established more than 50 years ago [1]. These criteria were chosen to identify women at high risk for the development of diabetes after pregnancy and not necessarily to identify pregnancies with increased risk for adverse perinatal outcome [2]. Two large randomised intervention trials have now demonstrated improvement in perinatal outcomes, especially in the frequency of large for gestational age (LGA) infants, in women who receive treatment for mild glucose intolerance during pregnancy [3, 4]. Progressively more data have shown that the risk of adverse perinatal outcomes is also associated with degrees of hyperglycaemia less severe than overt diabetes during pregnancy. The Hyperglycaemia and Adverse Pregnancy Outcome (HAPO) study showed a continuous and graded relationship between maternal hyperglycaemia and risk for adverse perinatal outcome, independent of other risk factors [5]. As there are no specific thresholds of hyperglycaemia at which the risk increases significantly, a consensus was necessary to decide on specific cut-offs to define new criteria for the diagnosis of GDM.

The prevalence of diabetes and obesity is increasing in the general population, where type 2 diabetes is often also undiagnosed [6]. Therefore, the incidence of type 2 diabetes is increasing in women of childbearing age, leading to more pregnant women with undiagnosed type 2 diabetes. In addition to diagnostic criteria for GDM, the timely diagnosis and treatment of pre-existing diabetes early in pregnancy is necessary as these women are at an increased risk for diabetes complications and congenital anomalies in their babies, as blood glucose levels are higher earlier in pregnancy. These women need treatment with hypoglycaemic agents such as insulin and close follow-up according to guidelines for women with pre-existing diabetes during pregnancy. Moreover, the management of diabetes needs to be continued after the delivery, similar to other women with known diabetes before pregnancy.

In June 2008, the International Association of Diabetes and Pregnancy Study Groups (IADPSG) organised a conference leading to a consensus statement for a new screening strategy and diagnosis of GDM [7]. The IADPSG advises screening for existing but unknown diabetes at the first prenatal clinic visit, especially in high-risk populations. Cut-offs for tests used to detect diabetes in the non-pregnant population (fasting plasma glucose $[\mathrm{FPG}] \geq 7.0 \mathrm{mmol} / \mathrm{l}$, random plasma glucose $\geq 11.1 \mathrm{mmol} / 1$ or $\mathrm{HbA}_{1 \mathrm{c}} \geq 6.5 \%$ [ $\left.47 \mathrm{mmol} / \mathrm{mol}\right]$ ) are recommended. If overt diabetes or GDM has not been diagnosed in early pregnancy or if the first antenatal contact is at 24 weeks' gestation or later, IADPSG advises that every woman should undergo a $75 \mathrm{~g} 2 \mathrm{~h}$ OGTT. The IADPSG Consensus Panel developed diagnostic cut-off points for FPG, 1 and $2 \mathrm{~h}$ plasma glucose measurements that conveyed an odds ratio for adverse outcomes of at least 1.75 compared with women with mean glucose levels in the HAPO study. More specifically, the cutoff points for the FPG, 1 and $2 \mathrm{~h}$ plasma glucose were chosen to reflect a $75 \%$ increase in risk based on the development of an LGA baby, an umbilical cord C-peptide in the offspring $>90$ th percentile and the percentage body fat in the offspring $>90$ th percentile. If the IADPSG had chosen the new criteria based on an odds ratio of 2.0 rather than 1.75 for the development of complications in the HAPO study, the threshold for abnormal values would be higher, and GDM prevalence in the HAPO study would have been lower. The IADPSG Consensus Panel considered different thresholds but decided by consensus to recommend the 1.75 risk threshold as the higher threshold would fail to identify many cases with nearly comparable risk of adverse outcomes.

The IADPSG now recommends a one-step diagnostic approach: one abnormal measurement from a $2 \mathrm{~h} 75 \mathrm{~g}$ OGTT is enough to diagnose GDM. These are the first diagnostic criteria for GDM based on perinatal outcome and are therefore unique. The IADPSG recommends the following glycaemic thresholds: $\mathrm{FPG} \geq 5.1 \mathrm{mmol} / \mathrm{l} ; 1 \mathrm{~h}$ plasma glucose $\geq 10 \mathrm{mmol} / \mathrm{l}$; and $2 \mathrm{~h}$ plasma glucose $\geq 8.5 \mathrm{mmol} / 1$ [7].

The IADPSG Consensus Panel now also recommends that $\mathrm{FPG} \geq 5.1 \mathrm{mmol} / \mathrm{l}$ in early pregnancy is classified as GDM [7]. This recommendation is debatable as it was reached mainly by consensus and uses the cut-off used for later pregnancy derived from the HAPO study. The ADA, the Australasian Diabetes in Pregnancy Society (ADIPS) and the Ministry of Health of China now recommend the implementation of the IADPSG screening strategy, but without endorsing the recommendation to classify $\mathrm{FPG} \geq 5.1 \mathrm{mmol} / \mathrm{l}$ in early pregnancy as GDM [8-10]. An evaluation of using FPG in the first prenatal visit to diagnose GDM in China showed that FPG between 6.1 and $6.9 \mathrm{mmol} / 1$ was a more accurate predictor of the development of GDM than FPG $\geq 5.1 \mathrm{mmol} / \mathrm{l}$. This study therefore does not support $\mathrm{FPG} \geq 5.1 \mathrm{mmol} / 1$ at the 
first prenatal visit as the criterion for GDM diagnosis in a Chinese population [11].

\section{Ongoing controversy around screening for gestational diabetes}

Internationally, the IADPSG consensus for diagnosing GDM remains controversial as, in most populations, it would lead to a very important increase in the reported prevalence of GDM [12]. Comments have been raised about the paucity of data on cost effectiveness of such a screening strategy, uncertainties about the clinical relevance of treating mild GDM based on the IADPSG criteria and the risk of progression from mild GDM to type 2 diabetes mellitus postpartum, and the lack of clinical controlled trials addressing the use of the IADPSG criteria [13]. The IADPSG criteria are partly based on surrogate outcomes, such as cord C-peptide. Moreover, the IADPSG recommendations focus on a glucocentric approach to decreasing adverse pregnancy outcomes, while a more integrated approach to defining risk, including other important risk factors such as ethnicity, family history, obesity and gestational weight gain, is needed to support work to reduce accelerated fetal growth. In addition, the diagnosis of GDM based on one abnormal value on the basis of only one test raises concern as the OGTT has poor reproducibility [13].

The main argument in favour of the new one-step diagnostic approach with stricter diagnostic criteria for GDM is that it will lead to opportunities for more women to receive a timely diagnosis and appropriate treatment for GDM. Adopting uniform diagnostic criteria for GDM will improve and encourage research in the domain of GDM. The use of a uniform $2 \mathrm{~h} 75 \mathrm{~g}$ OGTT in both the pregnant and non-pregnant population will also lead to an important simplification of laboratory results.

So far, two studies have addressed the cost effectiveness of GDM screening according to the IADPSG criteria using decision-analysis models for a US population [14, 15]. These have yielded conflicting results. One study showed that the IADPSG recommendations are cost effective only when post-delivery care reduces diabetes incidence [14]. In the second study, management based on the IADPSG criteria would be effective if treatment decreased the incidence of preeclampsia by $>0.55 \%$ and Caesarean delivery by $>2.7 \%$ [15]. Recent modelling based on data from the ATLANTIC Diabetes in Pregnancy (DIP) study shows that GDM plays an independent role in explaining variations in rates of emergency Caesarean section, neonatal unit admission and costs of care, and thus places a substantial economic burden on maternity care services [16].

While the ADA has, since December 2010, adopted the IADPSG recommendations, the American College of
Gynecologists and Obstetricians (ACOG) and an independent expert panel assigned by the National Institutes of Health (NIH) continue to promote the use of a two-step screening strategy: a universal screening strategy with the non-fasting $50 \mathrm{~g}$ glucose challenge test (GCT) followed by, if abnormal, a 3 h 100 g OGTT using the Carpenter and Coustan diagnostic criteria $[17,18]$. The panel was particularly concerned that the adoption of the IADPSG criteria would increase the prevalence of GDM and the corresponding costs and interventions, without clear demonstration of improvements in the most clinically important health and patient-centred outcomes.

In 2013 both the WHO and the Endocrine Society revised their guidelines and now advise that the IADPSG criteria should be used for the diagnosis of GDM $[19,20]$. In the current proposal, the new diagnostic criteria for GDM are therefore now called the new WHO criteria for GDM. In line with the IADPSG Consensus Panel recommendation, the WHO and the Endocrine Society also endorse the recommendation to classify an $\mathrm{FPG} \geq 5.1 \mathrm{mmol} / \mathrm{l}$ in early pregnancy as GDM. The WHO, however, does not consider $\mathrm{HbA}_{1 \mathrm{c}}$ for the diagnosis of overt diabetes.

The latest 2014 ADA recommendations specify that further research is needed to establish a uniform approach to diagnosing GDM and leave open the options of using the one-step IADPSG recommendation or a two-step screening strategy [21]. The WHO acknowledges that the quality of evidence for adapting the IADPSG recommendations is very low when evaluated using the GRADE system of ranking evidence strength [19]. Both WHO and the panel assigned by the NIH indicate that their positioning will be revised when new evidence is available.

\section{An EBCOG initiative to develop a proposal for the use of uniform diagnostic criteria for GDM in Europe}

Recent surveys demonstrate the strong variability internationally in screening, diagnosis and treatment of women with GDM [22]. A recent review also shows that screening and management of GDM is very variable across Europe, partly due to the ongoing controversy and inadequate clinician awareness of GDM [23]. A recent survey performed in the northern part of Belgium highlights that policies are often also inconsistent even within one region [24]. Comparisons between countries are very challenging because of the different screening strategies and diagnostic criteria used and different ethnicities. An important factor leading to this large variation in practice is probably the lack of consensus between many local and international scientific professional organisations.

Several countries in Europe such as Ireland, France and the French-speaking part of Belgium, have started to use a $75 \mathrm{~g}$ OGTT with IADPSG criteria, but often this is performed only 
in high-risk women and not universally [24-27]. In some countries in Europe, such as Germany, a 75 g OGTT with IADPSG criteria is not performed as a one-step screening strategy but instead a $75 \mathrm{~g}$ OGTT is performed only following an abnormal GCT [28]. However, there are currently no data on the validity of GCT as a universal screening test in a twostep strategy with IADPSG criteria. Some countries and regions in Europe use selective screening with the previous WHO diagnostic criteria (the UK and the Netherlands, for example), while others use the ACOG screening strategy (the Flemish part of Belgium, for example) [24, 29, 30]. Other countries use risk-factor-based or universal screening and the criteria of the EASD or the National Diabetes Data Group [31, 32]; see Text box: Commonly used screening strategies for GDM in Europe.

\section{Commonly used screening strategies for GDM in Europe \\ Universal screening \\ - 75 g OGTT with the new WHO criteria [19] \\ - $50 \mathrm{~g} \mathrm{GCT}$; if abnormal, followed by \\ - $75 \mathrm{~g}$ OGTT with the new WHO or Carpenter and \\ Coustan criteria [2], or \\ - 100 g OGTT with Carpenter and Coustan or the \\ National Diabetes Data Group criteria [2] \\ - Random glycaemia; if elevated, followed by $75 \mathrm{~g}$ \\ or $100 \mathrm{~g}$ OGTT}

Selective screening of high-risk groups with $75 \mathrm{~g}$ OGTT using one of the following:

- Previous WHO criteria

- New WHO criteria [19]

- Carpenter and Coustan criteria [2]

- EASD criteria

- Locally used criteria

Developing a consensus on screening for GDM in Europe is challenging, as ethnic populations are diverse across Europe and healthcare delivery systems differ. Many national societies have recently revised, or will soon revise, their guidelines after the new WHO recommendations. Most national societies in Europe do not recommend a universal one-step diagnostic approach with an OGTT, in part because of the associated increased workload and costs. The EBCOG has responded to this challenge by appointing a steering committee to prepare a proposal for the use of uniform diagnostic criteria for GDM in Europe. The steering committee includes members of the EBCOG and the Diabetic Pregnancy Study Group (DPSG) associated with the EASD, although this opinion paper is not an official statement from the DPSG. The co-authors P. Damm and R. Corcoy were also members of the IADPSG consensus group. The first meeting of the steering committee was held at the DPSG meeting in Malta in October 2013. The first version of the proposal was completed by December 2013 and then further adapted according to feedback from members of the steering committee. The proposal was reviewed by the national societies from April 2014, and was approved by the Council of the EBCOG at the end of November 2014.

\section{Proposal for screening for overt diabetes early in pregnancy}

As the prevalence of obesity and type 2 diabetes is also increasing in women of childbearing age in Europe, the use of an easy screening test will lead to an earlier diagnosis and treatment of overt diabetes. The proposal recommends screening for overt diabetes, particularly in high-risk groups (see Text box: Risk factors for diabetes and GDM), at preconception or the first antenatal contact using the cut-off values for diabetes outside pregnancy. As there is no clear evidence about which test performs the best, it is not feasible to recommend a single test to use exclusively. An FPG test is simple and can be done at a relatively low cost. It has also been shown that higher first-trimester fasting glucose levels, within what is currently considered a non-diabetic range, increase the risk of adverse pregnancy outcomes [33]. An FPG is more sensitive than $\mathrm{HbA}_{1 \mathrm{c}}$ for the diagnosis of diabetes. A random plasma glucose value has the lowest diagnostic sensitivity [34]. The higher cost of an $\mathrm{HbA}_{1 \mathrm{c}}$ test compared with an FPG test is a disadvantage, but the between- and within-individual coefficients of variation are substantially lower for $\mathrm{HbA}_{1 \mathrm{c}}$ than for FPG. $\mathrm{HbA}_{1 \mathrm{c}}$ testing also has the advantage that it can be performed without fasting. $\mathrm{HbA}_{1 \mathrm{c}}$ testing has now been standardised worldwide and most methods can be used with the most frequent haemoglobin traits. The altered red cell turnover in pregnancy means that $\mathrm{HbA}_{1 \mathrm{c}}$ cannot be used to diagnose GDM, but the test is accurate enough to use for the diagnosis of diabetes. The measurement of glycosuria alone is not advised as this has a very low sensitivity and is also often falsely positive in pregnancy [35].

\section{Risk factors for diabetes and GDM}

- Previous gestational diabetes

- Overweight/obesity

- Family history of diabetes (first-degree relative with diabetes)

- Previous macrosomia (infant birthweight $>4,000 \mathrm{~g}$ or $>90$ th percentile)

- Polycystic ovary syndrome

- Ethnicity: Mediterranean, South Asian, African black, North African, Caribbean, Middle Eastern, Hispanic 
It is generally considered that there is not enough evidence to recommend screening for GDM before 24-28 weeks of gestation. The IADPSG Consensus Panel recommends that FPG $\geq 5.1 \mathrm{mmol} / 1$ in early pregnancy is classified as GDM [7]. This is, however, debated as this recommendation was merely based on data extrapolated from the cut-off value used for the $75 \mathrm{~g}$ OGTT later in pregnancy. As evidence is lacking about both the women who would benefit most from screening and treatment of GDM before 24 weeks of pregnancy and the diagnostic approach that should be used for GDM, no clear recommendations can yet be made on the diagnostic criteria for GDM in early pregnancy.

Controlling hyperglycaemia in pregnancy is certainly not the only essential factor for reducing the number of LGA newborns. This is confirmed by analyses of the HAPO study showing that both maternal GDM and obesity are independently associated with adverse pregnancy outcomes such as LGA, pre-eclampsia, Caesarean section and newborn percentage body fat $>90$ th percentile and that the combination of obesity and GDM confers a greater risk of these adverse pregnancy outcomes than either obesity or GDM alone [36]. Maternal obesity is also a major independent risk factor for gestational hypertension, congenital abnormalities and birth trauma [37]. A recent meta-analysis has shown that lifestyle intervention in obese pregnant women reduces maternal weight gain, but without differences in rates of Caesarean section, LGA or macrosomia [38]. Gestational weight gain is also a known independent risk factor for accelerated fetal growth [39]. An integrated approach is therefore necessary to define and treat all the risk factors inducing fetal energy excess and accelerated fetal growth. In line with the ACOG recommendation, the current proposal is therefore that preconception assessment and counselling are strongly encouraged for overweight and obese women and should include the provision of specific information concerning the maternal and fetal risks of obesity in pregnancy, as well as encouragement to undertake a weight-reduction programme [40]. To avoid excessive weight gain during pregnancy (according to the Institute of Medicine guidelines), the recommendation is now also to offer lifestyle counselling to every woman early in pregnancy [41].

\section{Proposal to use the new WHO diagnostic criteria for GDM in Europe at 24-28 weeks of pregnancy}

The main discussion now is around the threshold at which hyperglycaemic treatment of GDM becomes cost effective $[42,43]$. There is no doubt that women with the highest degree of hyperglycaemia should be treated and that screening is highly valuable in this group. Treatment of milder degrees of hyperglycaemia remains controversial as the potential advantages of diagnosis and treatment might not outweigh the disadvantages. In addition to the costs for organising screening and the inconvenience of performing a test that requires a pregnant woman to fast, the main disadvantage related to a generalised one-step diagnostic approach is that more intensive diagnosis and treatment might also result in an increased medicalisation of antenatal care. This might also divert attention from women with established diabetes who are likely to benefit most. A universal one-step diagnostic approach implies that all pregnant women should receive an OGTT, which might be logistically challenging in some centres and will certainly lead to a substantial increase in healthcare costs. Exploring shared-care protocols with testing distributed in multiple laboratories might help to better organise the process. It is important, however, that the plasma glucose analyses are always performed using a gold-standard method. Performing OGTTs in primary care might also be an option, but may be difficult to organise; home OGTT kits are also coming to the market.

No intervention trials have been performed using the new WHO criteria, but two large intervention trials have shown that treatment of mild forms of GDM is associated with decreased rates of fetal overgrowth and pre-eclampsia $[3,4]$. There was substantial overlap between the glucose values used for inclusion in these randomised controlled trials and those recommended by the IADPSG Consensus Panel. Moreover, a recent Spanish cohort study showed that, compared with the previous two-step screening strategy using the Carpenter and Coustan criteria, the one-step approach with the IADPSG criteria was associated with reduced rates of adverse pregnancy outcomes and was also cost effective [44]. The IADPSG criteria are the first diagnostic criteria for GDM based on perinatal outcome and are unique. The EBCOG proposal is in line with the recent WHO recommendation to use the $75 \mathrm{~g}$ OGTT with the new WHO diagnostic criteria for GDM at 24-28 weeks of pregnancy (Fig. 1). The use of the new diagnostic criteria for GDM will lead to an opportunity for more women to be diagnosed in a timely fashion and receive appropriate and timely treatment for GDM. The use of a uniform $2 \mathrm{~h} 75 \mathrm{~g}$ OGTT with the same diagnostic criteria in pregnancy will lead to an important simplification of approach and facilitate research in GDM across European countries. The use of the same diagnostic criteria for GDM across Europe will be an important first step towards uniformity in Europe.

However, more research is needed to evaluate screening strategies across different populations in Europe, and determine which would be the best. Therefore, no clear recommendations have been made on whether a universal one-step, twostep or a risk-factor-based screening approach should be used. More data are needed on the cost effectiveness of a universal one-step diagnostic approach with the $75 \mathrm{~g}$ OGTT and the new WHO criteria compared with other screening approaches for GDM in European populations. The new diagnostic WHO criteria for GDM should be evaluated in diverse socioeconomic settings and different ethnic groups across Europe. In some ethnic groups a two-step screening strategy with an FPG 


\section{Conclusion}

Because screening, diagnostic criteria and treatment of GDM are highly inconsistent across Europe, the development of a uniform diagnostic approach for GDM is necessary. This will give the opportunity for more women to receive a timely diagnosis and appropriate treatment for GDM. Implementing uniform diagnostic criteria for GDM will also improve and encourage research in the domain of GDM.

The current proposal is to screen for overt diabetes at preconception or at the first prenatal contact, using the cut-off values for diabetes outside pregnancy; screening should focus particularly on high-risk groups. When screening for GDM is performed at 24 weeks' gestation or later, the $2 \mathrm{~h} 75 \mathrm{~g}$ OGTT should be used with the new WHO diagnostic criteria for GDM. However, more research is needed to determine the most effective screening strategy across different populations in Europe. Therefore, no clear recommendation can be made on whether a universal one-step, two-step or risk-factor-based screening approach should be used. The use of the same diagnostic criteria for GDM across Europe will be an important first step towards uniformity.

Acknowledgements $\mathrm{KB}$ is the recipient of a Clinical Doctoral Scholarship of 'FWO Vlaanderen' and RD is the recipient of a 'Fundamenteel Klinisch Navorserschap FWO Vlaanderen'. FD is the recipient of a number of grants supporting research in GDM from the Irish Health Research Board.

Funding The development of the proposal was not funded.

Duality of interest The authors declare that there is no duality of interest associated with this manuscript

Contribution statement All authors contributed to the concept of the proposal. KB drafted the article and all authors revised it critically for important intellectual content. All authors approved the final version to be published. $\mathrm{KB}$ is the guarantor of this work.

\section{References}

1. O'Sullivan JB, Mahan CM (1964) Criteria for oral glucose tolerance test in pregnancy. Diabetes 13:278-285

2. Metzger BE, Buchanan TA, Coustan DR et al (2007) Summary and recommendations of the Fifth International Workshop-Conference on Gestational Diabetes Mellitus. Diabetes Care 30(Suppl 2):S251S260

3. Crowther CA, Hiller JE, Moss JR et al (2005) Australian Carbohydrate Intolerance Study in Pregnant Women (ACHOIS) Trial Group. Effect of treatment of gestational diabetes mellitus on pregnancy outcomes. N Engl J Med 352:2477-2486

4. Landon MB, Spong CY, Thom E et al (2009) A multicenter, randomized trial of treatment for mild gestational diabetes. N Engl J Med 361:1339-1348

5. HAPO Study Cooperative Research Group, Metzger BE, Lowe LP, Dyer AR et al (2008) Hyperglycemia and adverse pregnancy outcomes. N Engl J Med 358:1991-2002
6. Lawrence JM, Contreras R, Chen W, Sacks DA (2008) Trends in the prevalence of preexisting diabetes and gestational diabetes mellitus among a racially/ethnically diverse population of pregnant women, 1999-2005. Diabetes Care 31:899-904

7. International Association of Diabetes and Pregnancy Study Groups Consensus Panel (2010) International association of diabetes and pregnancy study groups recommendations on the diagnostic and classification of hyperglycemia in pregnancy. Diabetes Care 33: 676-682

8. American Diabetes Association (2013) Standards of medical care in diabetes-2013. Diabetes Care 36:S11-S66

9. Nankervis A, McIntyre HD, Moses R, et al for the Australasian Diabetes in Pregnancy Society (2013) ADIPS consensus guidelines for the testing and diagnosis of gestational diabetes mellitus in Australia. Available from www.adips.org/downloads/ A D I P S Cons en sus Guide 1 in e s G D M - 03.05 . 13VersionACCEPTEDFINAL.pdf. Accessed 17 Nov 2013

10. Zhu W-W, Yang H-X (2013) Diagnosis of gestational diabetes mellitus in China. Diabetes Care 36, e76

11. Zhu W-W, Yang H-X, Wei Y-M et al (2013) Evaluation of the value of fasting plasma glucose in first prenatal visit to diagnose gestational diabetes mellitus in China. Diabetes Care 36:586-590

12. Jenum AK, Mǿrkrid K, Sletner L et al (2012) Impact of ethnicity on gestational diabetes identified with the WHO and the modified IADPSG criteria: a population-based cohort study. Eur J Endocrinol 166:317-324

13. Cundy T, Ackermann E, Ryan EA (2014) Gestational diabetes: new criteria may triple the prevalence but effect on outcomes is unclear. BMJ 348:g1567

14. Werner EF, Petther CM, Zuckerwise L et al (2012) Screening for gestational diabetes mellitus: are the criteria proposed by the International Association of Diabetes and Pregnancy Study Group cost-effective? Diabetes Care 35:529-535

15. Mission JF, Ohno MS, Cheng YW, Caughey AB (2012) Gestational diabetes screening with new IADPSG guidelines: a costeffectiveness analysis. Am J Obstet Gynecol 207:326e1-326e9

16. Gillespie P, Cullinan J, O'Neil C, Dunne F (2013) Modeling the independent effects of gestational diabetes mellitus on maternity care and costs. Diabetes Care 36:1111-1116

17. Committee on Obstetric Practice (2013) Practice bulletin no. 137: gestational diabetes mellitus. Obstet Gynecol 122:406-416

18. Vandorsten JP, Dodson WC, Espeland MA et al (2013) NIH consensus development conference: diagnosing gestational diabetes mellitus. NIH Consens State Sci Statements 29:1-31

19. The World Health Organization guideline 2013 (2013) Diagnostic criteria and classification of hyperglycaemia first detected in pregnancy. Available from http://apps.who.int/iris/bitstream/10665/ 85975/1/WHO_NMH_MND_13.2_eng.pdf. Accessed 17 Nov 2013

20. Blumer I, Hadar E, Hadden DR et al (2013) Diabetes and pregnancy: an Endocrine Society clinical practice guideline. J Clin Endocrinol Metab 98:4227-4249

21. American Diabetes Association (2014) Standards of medical care in diabetes. Diabetes Care 37:S14-S80

22. Jiwani A, Marseille E, Lohse N, Damm P, Hod M, Kahn JG (2012) Gestational diabetes mellitus: results from a survey of country prevalence and practices. J Matern Fetal Neonatal Med 25:600-610

23. Buckley BS, Harreiter J, Damm P et al (2012) Gestational diabetes mellitus in Europe: prevalence, current screening practice and barriers to screening. A review. Diabet Med 29:844-854

24. Benhalima K, Van Crombrugge P, Devlieger R et al (2013) Screening for pregestational and gestational diabetes in pregnancy: a survey of obstetrical centers in the northern part of Belgium. Diabetol Metab Syndr 5:66

25. Vanderijst J-F, Debieve F, Doucet F et al (2012) Stratégie de dépistage et critères diagnostiques du diabète gestationnel. 
Propositions du GGOLFB. Rev Med Liege 67(4):179-185, [article in French]

26. Le Collège national des gynécologues et obstétriciens français et par la Société francophone du diabète (2010) Recommendations pour la pratique Clinique: le diabète gestationnel. Available from www.cngof.asso.fr/D_TELE/RPC_DIABETE_2010.pdf. Accessed 19 Nov 2013 [article in French]

27. Health Service Executive of Ireland (2010) Guidelines for the management of pre-gestational and gestational diabetes mellitus from pre-conception to the postnatal period. Available from www.hse.ie/ eng/services/Publications/corporate/NursingMidwifery\% 20Services/onsdguidelinesgestationaldiabetes.pdf. Accessed 19 Nov 2013

28. Praxisleitlinie der Deutschen Diabetes-Gesellschaft (DDG) und der Deutschen Gesellschaft für Gynäkologie und Geburtshilfe (DGGG) (2012) Gestationsdiabetes mellitus (GDM) -Diagnostik, Therapie u. Nachsorge. Available from www.deutsche-diabetes-gesellschaft. de/fileadmin/Redakteur/Leitlinien/Praxisleitlinien/2012/DuS S212_Praxisempfehlungen_Kleinwechter-etal_GDM_S174-184.pdf. Accessed 19 Nov 2013 [article in German]

29. National Institute for Health and Clinical Excellence (2008) Diabetes in pregnancy: management of diabetes and its complications from pre-conception to the postnatal period. Available from www.nice.org.uk/nicemedia/pdf/cg063guidance.pdf. Accessed 19 Nov 2013

30. Richtlijn van de Nederlands Vereniging voor Obstetrie en Gynaecologie (2010) Diabetes mellitus en zwangerschap. Available from http://nvog-documenten.nl/index.php?pagina=/ site/pagina.php\&id=54321. Accessed 19 Nov 2013 [article in Dutch]

31. Houshmand A, Møller Jensen D, Mathiesen ER, Damm P (2013) Evolution of diagnostic criteria for gestational diabetes mellitus. Acta Obstet Gynecol Scand 92:739-745

32. Corcoy R, Lumbreras B, Bartha JL, Ricart W, Grupo Español de Diabetes y Embarazo (2010) New diagnostic criteria for gestational diabetes mellitus after the HAPO study. Are they valid in our environment? Endocrinol Nutr 57:277-280

33. Riskin-Mashiah S, Younes G, Dati A, Auslender R (2009) Firsttrimester fasting hyperglycemia and adverse pregnancy outcomes. Diabetes Care 32:1639-1643

34. The international Expert Committee (2009) International Expert Committee Report on the role of the Alc assay in the diagnosis of diabetes. Diabetes Care 32:1327-1334

35. Buhling KJ, Elze L, Henrich W et al (2004) The usefulness of glycosuria and the influence of maternal blood pressure in screening for gestational diabetes. Eur J Obstet Gynecol Reprod Biol 113: 145-148

36. Catalano PM, McIntyre HD, Cruickshank JK et al (2012) The hyperglycemia and adverse pregnancy outcome study: associations of GDM and obesity with pregnancy outcomes. Diabetes Care 35: 780-786
37. Ricart W, López J, Mozas J et al (2005) Spanish Group for the Study of the Impact of Carpenter and Coustan GDM Thresholds. Body mass index has a greater impact on pregnancy outcomes than gestational hyperglycaemia. Diabetologia 48: $1736-1742$

38. Oteng-Ntim E, Varma R, Croker H, Poston L, Doyle P (2012) Lifestyle interventions for overweight and obese pregnant women to improve pregnancy outcome: systematic review and metaanalysis. BMC Med 10:47

39. Ludwig DS, Currie J (2010) The association between pregnancy weight gain and birthweight: a within-family comparison. Lancet 376:984-990

40. American College of Obstetricians and Gynecologists (2013) ACOG Committee opinion no. 549: obesity in pregnancy. Obstet Gynecol 121:213-217

41. Committee to Reexamine IOM Pregnancy Weight Guidelines, Food and Nutrition Board,Board on Children et al (2009) In: Rasmussen KM, Yaktine AL (eds) Weight gain during pregnancy: reexamining guidelines. The National Academies Press, Washington, DC

42. Ryan EA (2011) Diagnosing gestational diabetes. Diabetologia 54: 480-486

43. Waugh N, Pearson D, Royle P (2010) Screening for hyperglycaemia in pregnancy: consensus and controversy. Best Pract Res Clin Endocrinol Metab 24:553-571

44. Duran A, Sáenz S, Rorrejón MJ et al (2014) Introduction of IADP SG criteria for the screening and diagnosis of gestational diabetes mellitus results in improved pregnancy outcomes at a lower cost in a large cohort of pregnant women: the St Carlos gestational diabetes study. Diabetes Care 37:2442-2450

45. Agarwal MM, Dhatt GS, Shah SM (2013) Gestational diabetes mellitus: simplifying the international association of diabetes and pregnancy diagnostic algorithm using fasting plasma glucose. Diabetes Care 33:2018-2020

46. Zhu WW, Fan L, Yang HX et al (2013) Fasting plasma glucose at 24-28 weeks to screen for gestational diabetes mellitus: new evidence from China. Diabetes Care 36:2038-2040

47. Savona-Ventura C, Vassallo J, Marre M, Karamanos B (2012) Hyperglycaemia in pregnancy in Mediterranean women. Acta Diabetol 49:473-480

48. Bellamy L, Casas JP, Hingorani AD, Williams D (2009) Type 2 diabetes mellitus after gestational diabetes: a systematic review and meta-analysis. Lancet 373:1773-1779

49. Reinblatt SL, Morin L, Meltzer SJ (2006) The importance of a postpartum $75 \mathrm{~g}$ oral glucose tolerance test in women with gestational diabetes. J Obstet Gynaecol Can 28:690-694

50. Picón MJ, Murri M, Muñoz A, Fernández-García JC, Gomez-Huelgas R, Tinahones FJ (2012) Hemoglobin A1c versus oral glucose tolerance test in postpartum diabetes screening. Diabetes Care 35:1648-1653 